of the ecosystem, which could be considered a benefit to all member organisms.

Owen and Wiegert (Oikos 27, 488; 1976) sought for more tangible benefits to plants from the existence of a contingent of grazers in their midst. They asked the question in the forcible form "Do consumers maximize plant fitness?", and their guarded but affirmative reply was based on some detailed illustrations from nature. For example, they use the figures of Llewellyn (J. appl. Ecol. 9, 261; 1972) to show that a population of a million aphids inhabiting a single lime (Tilia) tree, $14 \mathrm{~m}$ in height, could release sugar as honeydew at the rate of $\mathbf{4 0 7} \mathrm{g}$ day $^{-1}$. Llewellyn's overall annual estimate of sugar rain was $1 \mathrm{~kg} \mathrm{~m}^{-2}$. This will obviously have a profound influence on soil microbial activity and Owen and Wiegert single out for attention the free-living nitrogen-fixing microbial genus Azotobacter. The energetic stimulation of this component of the soil flora could result in higher rates of nitrogen input to the soil and consequently the ultimate encouragement of growth in the vegetation. This assumes, of course, that Azotobacter will be the sole or main beneficiary from the aphidic manna, which is not necessarily so, since the ecology of the competitive interactions among soil microbes is likely to be complex, but it is nevertheless reasonable to suppose that some increase in nitrogen fixation will occur. The important question is whether the nitrogen gain to the plant is worth the carbon loss.

A still more direct example of a stimulating consumer has been provided by Boscher (Oikos 33, 451; 1979) in her studies of the cultivated leek, Allium porrum. This plant is frequently blighted by the leek moth Acrolepiopsis assectella, the larvae of which feed on leaves, scapes and pedicels, though not normally on the flowers, which Arnault (C. hebd. Acad. Sci Paris 280, 2487; 1975) considered to be toxic to them. Boscher found that moderate defoliation of the leek had no adverse effect on fruit production, indeed some plants produced more fruit. Severe defoliation resulted in a considerable development of vegetative bulbils in the inflorescence. This is a reproductive strategy which allows rapid expansion of the population, since the bulbils produce plants which are sexually mature in their first year whereas plants grown from seed only flower in their second year. The switch to vegetative propagation is thus controlled by the consumer organism.

There are, of course, some plants which have themselves turned predator; some fungi trap and consume detritivorous arthropods, and there are the insectivorous pitcher-plants and sundews. But a more subtle system for the exploitation of animals as an energy resource has been developed by some tropical epiphytes which have formed associations with ants. These were first described back in the

\title{
The parity of the pion
}

\section{from John Edgington}

TEXTBOOKS of nuclear physics are not noted for their flair and style. A rapidly developing subject, using difficult theoretical techniques to interpret and codify great quantities of experimental data, nuclear physics rarely affords us glimpses of the essential simplicity of nature, and the standard pedagogy tends to reflect this. However a result recently obtained by C. Wilkin (J. Phys. G, L5, 1980 ), though not new, is so refreshingly simple in its derivation that it should rapidly find its place in undergraduate courses. The topic is the intrinsic parity of the $\pi$-meson.

The original (and so far only) determination of the pion's parity was that made by Panofsky at Berkeley in 1951 , confirmed in 1954 by Steinberger at Columbia. Drawing on an idea of Ferretti dating from 1946, the argument has been outlined in many textbooks since, notably in Bethe and the Hoffmann's semi-classic 'Mesons and Fields'. The reaction studied is $\pi^{-}+d \rightarrow 2 n$; arguing from general conservation principles one shows that the very existence of this reaction requires the pion to have negative parity. The reasoning is unambiguous but requires knowledge of the initial quantum state of the $\pi^{-d}$ system; the argument is that the pion cascades down through its electron-like orbits until the final strong interaction occurs in the lowest $(l=0)$ orbit. This assumption, though highly plausible, is not absolutely compelling. That the pion's parity is negative is not seriously in doubt, but it would be comforting to have a slightly more watertight experimental proof.

Wilkin has provided such a proof. $\mathrm{He}$ uses some results obtained at SIN, Switzerland, by a group led by R. Hess of Geneva University, and reported to the 8th ICOHEPANS* Conference at

eighteenth century, but have recently been examined more closely by Janzen (Biotropica 6, 237; 1974) and Huxley (New Phytol. 80, 231; 1979). The stems of these plants are swollen and become hollow as a result of tissue death early in the life of the plant. The inner surfaces of these cavities are covered with warty protrusions. The hollow stems are invariably occupied by ants, and often by a variety of other organisms. The ants deposit quantities of detritus within these cavities and it was suggested during the last century that this could provide a source of inorganic elements for the plants if they were able to absorb them.

In laboratory and field experiments on two of these ant plant species, Myrmecodia and Hydnophytum, Huxley found that, in the presence of ants or of added inorganic nutrients, the plants grew more rapidly
Vancouver last August. Hess et al. have been studying the reaction $\mathrm{p}+\mathrm{p} \rightarrow \pi^{+}+\mathrm{d}$, using a polarised beam and target; they have measured the dependence of the cross section on the relative spin orientations of target and projectile. Their motivation was to provide further data on the spin-dependence of the nucleon-nucleon interaction, and the present result is an amusing and unexpected bonus.

Wilkin points out that, in the forward direction, there is a simple relation between two coefficients, $A_{L L}$ and $A_{T T}$, both of which measure the difference in reaction probability between states whose proton spins are aligned parallel, and anti-parallel, to one another. $A_{L L}$ refers to the case where the spins are directed parallel (or anti-parallel) to the projectile's motion, while $A_{\text {TT }}$ refers to the case where the spins are at right angles to this. Wilkin shows that

$$
A_{L L} \pm 2 A_{T T}=1
$$

the positive (negative) sign being taken if the pion has positive (negative) parity. By making a small extrapolation of the data of Hess et al. to the forward direction, he finds $A_{L L}=-0.3, A_{T T}=-0.6$; these values, with their rather small uncertainties, conform very comfortably with the notion that the parity of the pion is negative. Positive parity is unambiguously ruled out. Since $A_{L L}$ may not exceed unity, positive parity would forbid $\mathbf{A}_{\mathrm{TT}}$ from being negative, but it manifestly is so. This simple, yet pleasing demonstration of the pseudoscalar nature of the pion seems to have no controversial aspects.

John Edgington is a lecturer in the Department of Physics, Queen Mary College, London.

*International Conference on High Energy Physics and Nuclear Structure.

than without these. When ants were fed on honey water containing ${ }^{32} \mathrm{P}$ as phosphate and ${ }^{35} \mathrm{~S}$ as sulphate and as methionine, subsequent autoradiography showed an accumulation of radioactivity around the surfaces of the warts and also in the plant vascular tissue. So the plant can absorb these elements, possibly by way of faecal material in the cavities. Rickson (Amer. J. Bot. 66,$87 ; 1979$ ) has conducted some similar experiments in which he provided the ant plant and its resident ants with a supply of fly larvae labelled with ${ }^{14} \mathrm{C}$. Within 6 to 8 weeks radioactivity could be detected throughout the plants, demonstrating absorption and translocation of organic material either directly from the decaying fly larvae or indirectly through ant faeces.

Rickson proposes that the ants donate a proportion of their food to the host plant, 